\title{
Experimentally Observed Dynamical Characteristics of Mutually Coupled Semiconductor Lasers With or Without Optoelectronic Feedback
}

\author{
S. Tang, M.C. Chiang, J.M. Liu, R. Vicente ${ }^{a}$, and C.R. Mirasso ${ }^{a}$ \\ Electrical Engineering Department, University of California, Los Angeles, Los Angeles, CA \\ 90095-1594, USA \\ ${ }^{a}$ On leave from Departament de Fisica, Universitat de les Illes Balears, E-07122 Palma de \\ Mallorca, Spain
}

\begin{abstract}
Mutually-coupled semiconductor lasers are of great current interest because of the important insight they provide into coupled physical, chemical, and biological systems. Two semiconductor lasers either with or without optoelectronic feedback are mutually coupled together through optoelectronic paths. It is found that mutual coupling can significantly affect the dynamics of the semiconductor lasers, depending on the coupling delay time and the coupling strength. Interesting phenomena such as generation of chaos, quasiperiodic and period-doubling bifurcation to chaos, and death by delay are observed. Synchronization of the chaotic outputs from mutually coupled semiconductor lasers is also observed.
\end{abstract}

Keywords: Chaos, Semiconductor Lasers, Mutual Coupling, Optoelectronic Feedback

\section{INTRODUCTION}

Chaotic optical communications with messages encoded in chaotic optical waveforms and decoded through chaos synchronization have been widely investigated and demonstrated using semiconductor lasers. ${ }^{1,2}$ Nonlinear dynamics of semiconductor lasers are of great interests because of the important roles semiconductor lasers play in such chaotic optical communications. Chaotic dynamics can be induced in a semiconductor laser by increasing the dynamical dimension of the laser through a proper external perturbation, such as optical feedback, optical injection, or optoelectronic feedback. With optoelectronic feedback, a single-mode semiconductor laser can have chaotic dynamics in certain operating conditions. Either positive ${ }^{3}$ or negative ${ }^{4}$ optoelectronic feedback can be applied to a solitary single-mode semiconductor laser to generate chaotic dynamics. In both cases, the laser follows a quasiperiodicity route to chaotic pulsing.

While the dynamics of individual semiconductor lasers have been widely investigated, the effects of coupling between two semiconductor lasers have been of great interests recently. In unidirectionally coupled semiconductor lasers, chaos synchronization and chaotic communications have been demonstrated. Semiconductor lasers can also be mutually coupled. ${ }^{5,6}$ Leading and lagged synchronization has been observed in semiconductor lasers with mutual optical coupling. Mutual coupling also induces new nonlinear dynamical phenomena and significantly changes the dynamics of uncoupled semiconductor lasers. Uncoupled semiconductor lasers are independent nonlinear oscillators. Mutual coupling connects those nonlinear oscillators together. Mutual coupling has been observed to stabilize and quench the oscillation amplitude such as in the phenomenon of death by delay. Mutual coupling can also destabilize the nonlinear system to generate highly complex chaos.

In this paper, we study the nonlinear dynamics of two semiconductor lasers that are mutually coupled through optoelectronic paths. Three different system configurations with the presence or absence of feedback to the lasers are investigated. The effect of mutual coupling on the semiconductor laser dynamics is found to be significant. Stabilization of oscillation is observed due to mutual coupling. Highly complex chaos and the route to chaos are also demonstrated. Synchronization of the dynamical outputs from the lasers with mutual coupling is also

Further author information: (Send correspondence to J.M. Liu)

J.M. Liu: E-mail: liu@ee.ucla.edu, Telephone: 1-310-206-2097 

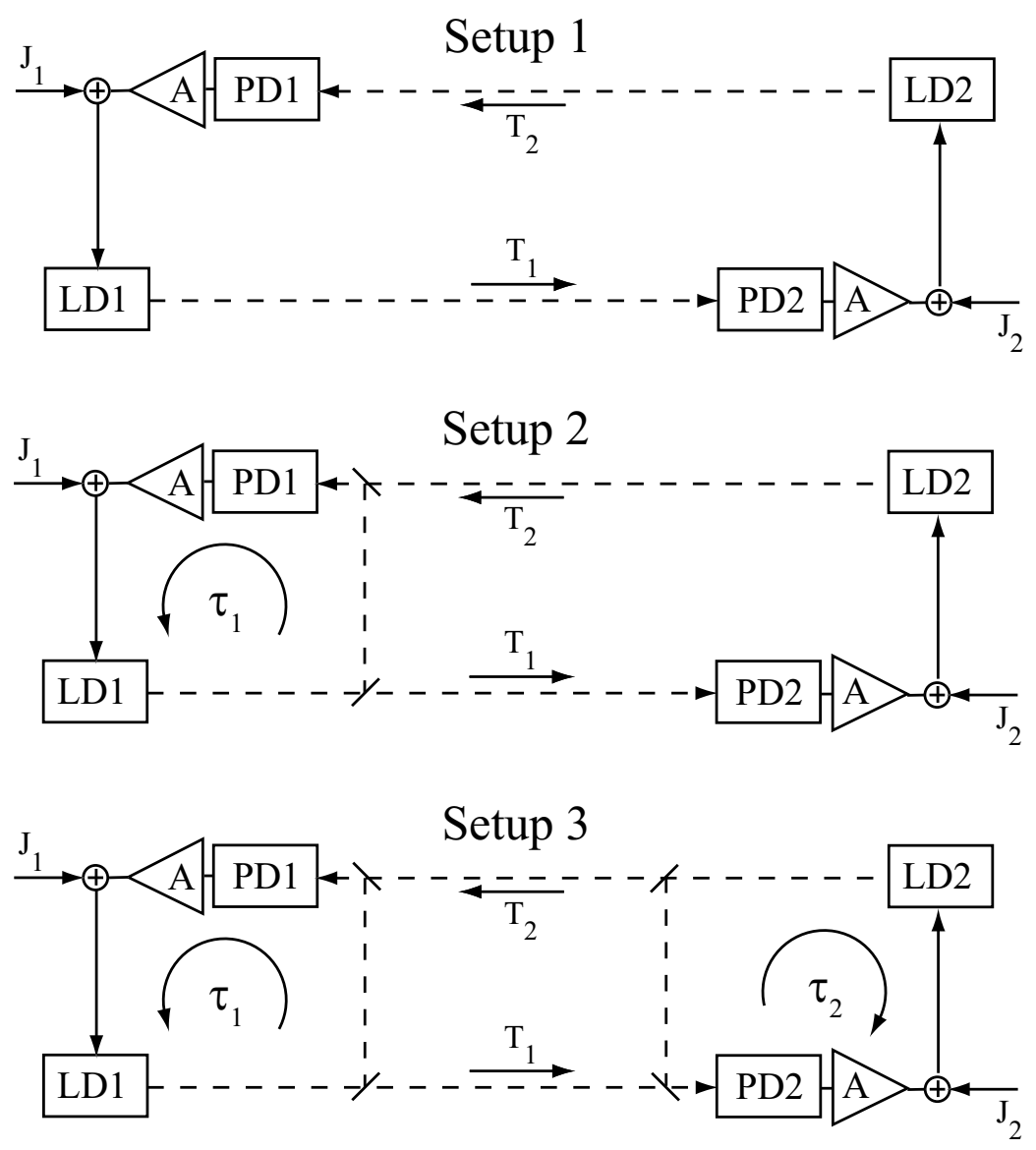

Figure 1. Schematics of mutually coupled semiconductor lasers. Setup 1, mutually coupled semiconductor lasers without optoelectronic feedback; Setup 2, mutually coupled semiconductor lasers with optoelectronic feedback for only one laser; Setup 3, mutually coupled semiconductor lasers with optoelectronic feedback for both lasers. LD: Laser diode; PD: Photodetector; A: Amplifier; $\tau$ : Feedback delay time; $T$ : Mutual coupling delay time; $J$ : Bias current.

studied. The organization of this paper is as follows: The system modeling and numerical results are given in Section 2. Sections 3, 4, and 5 cover the experimentally observed effects of mutual coupling for each of the three different system setups. Various dynamical states under different operation conditions for different coupling configurations are discussed in Section 6. A brief conclusion is given in Section 7.

\section{SYSTEM CONFIGURATION AND MODELING}

The schematics of semiconductor lasers with mutual optoelectronic coupling are shown in Fig. 1. Depending on the presence or absence of optoelectronic feedback to the lasers, the system can have three different configurations. In all three configurations, the two semiconductor lasers are mutually coupled. The output of laser diode 1 (LD1) is coupled to laser diode 2 (LD2) through an optoelectronic path which is consisted of photodetector 2 (PD2) and an amplifier. The detector PD2 converts the optical signal into an electronic signal. The electronic signal after PD2 and the amplifier is sent to LD2 through its current drive. Similarly, the output of LD2 is coupled to LD1 through another optoelectronic path consisting of photodetector 1 (PD1) and an amplifier. The differences in the setups are in the optoelectronic feedback. In Setup 1, no laser has any optoelectronic feedback. In Setup 2, only one laser has optoelectronic feedback. In Setup 3, both lasers have optoelectronic feedback. Under certain conditions, optoelectronic feedback can drive a semiconductor laser into nonlinear oscillation, such as regular pulsing, quasiperiodic pulsing, or chaotic pulsing. The rich nonlinear dynamics of semiconductor lasers with 
optoelectronic feedback have been demonstrated. ${ }^{3,4}$ In this paper, we study the coupling effect on semiconductor lasers with mutual optoelectronic coupling.

The two semiconductor lasers can be modeled by the rate equations of the intracavity photon density, $S$, and the carrier density, $N$. LD1 can be modeled as

$$
\begin{aligned}
\frac{d S_{1}}{d t} & =-\gamma_{\mathrm{c} 1} S_{1}+\Gamma g_{1}\left(N_{1}, S_{1}\right) S_{1}+2 \sqrt{S_{0} S_{1}} F_{\mathrm{s} 1} \\
\frac{d N_{1}}{d t} & =\frac{J_{1}}{e d}\left[1+\xi_{\mathrm{f} 1} y_{\mathrm{f} 1}\left(t-\tau_{1}\right)+\xi_{\mathrm{c} 1} y_{\mathrm{c} 1}\left(t-T_{2}\right)\right]-\gamma_{\mathrm{s} 1} N_{1}-g_{1}\left(N_{1}, S_{1}\right) S_{1}, \\
y_{\mathrm{f} 1}(t) & =\int_{-\infty}^{t} d \eta f_{1}(t-\eta) S_{1}(\eta) / S_{0}, \\
y_{\mathrm{c} 1}(t) & =\int_{-\infty}^{t} d \eta f_{1}(t-\eta) S_{2}(\eta) / S_{0} .
\end{aligned}
$$

The signal $\xi_{\mathrm{f} 1} y_{\mathrm{f} 1}\left(t-\tau_{1}\right)$ in Eq. (2) is the feedback signal of LD1, where $\xi_{\mathrm{f} 1}$ is the feedback strength and $\tau_{1}$ is the feedback delay time. As is shown in Eq. $(3), y_{\mathrm{f} 1}(t)$ is the convolution of $S_{1}(t)$ with the frequency response function $f_{1}(t)$ of the photodetector and the amplifier in the loop of LD1. Meanwhile, the signal $\xi_{\mathrm{c} 1} y_{\mathrm{c} 1}\left(t-T_{2}\right)$ is the coupling signal from LD2 to LD1, where $\xi_{\mathrm{c} 1}$ is the coupling strength and $T_{2}$ is the coupling delay time. As is shown in Eq. (4), $y_{\mathrm{c} 1}(t)$ is the convolution of $S_{2}(t)$ with the frequency response function $f_{1}(t)$. The parameter $\xi_{\mathrm{f} 1}$ or $\xi_{\mathrm{c} 1}$ respectively goes to zero if there is no optoelectronic feedback or coupling to LD1. In the configuration of Fig. 1, both the feedback and the coupling signals are bandwidth-limited by the frequency response function $f_{1}(t)$ of the photodetector and the amplifier in the loop of LD1.

Similarly, LD2 can be modeled as

$$
\begin{aligned}
\frac{d S_{2}}{d t} & =-\gamma_{\mathrm{c} 2} S_{2}+\Gamma g_{2}\left(N_{2}, S_{2}\right) S_{2}+2 \sqrt{S_{0} S_{2}} F_{\mathrm{s} 2} \\
\frac{d N_{2}}{d t} & =\frac{J_{2}}{e d}\left[1+\xi_{\mathrm{f} 2} y_{\mathrm{f} 2}\left(t-\tau_{2}\right)+\xi_{\mathrm{c} 2} y_{\mathrm{c} 2}\left(t-T_{1}\right)\right]-\gamma_{\mathrm{s} 2} N_{2}-g_{2}\left(N_{2}, S_{2}\right) S_{2}, \\
y_{\mathrm{f} 2}(t) & =\int_{-\infty}^{t} d \eta f_{2}(t-\eta) S_{2}(\eta) / S_{0} \\
y_{\mathrm{c} 2}(t) & =\int_{-\infty}^{t} d \eta f_{2}(t-\eta) S_{1}(\eta) / S_{0}
\end{aligned}
$$

The corresponding parameters in LD2 have the same meaning as those in LD1. The parameter $\xi_{\mathrm{f} 2}$ or $\xi_{\mathrm{c} 2}$ respectively goes to zero if there is no optoelectronic feedback or coupling to LD2. Other parameters in the rate equations are the free-running intracavity photon density $S_{0}$ when the laser is not subject to feedback, the optical gain coefficient $g(N, S)$ as a function of $N$ and $S$, the bias current density $J$, the cavity photon decay rate $\gamma_{\mathrm{c}}$, the spontaneous carrier decay rate $\gamma_{\mathrm{s}}$, the confinement factor of the laser waveguide $\Gamma$, the electronic charge constant $e$, the active layer thickness $d$, and the stochastic noise term $F_{\mathrm{s}}$.

The effect of coupling on the semiconductor lasers can be controlled by the four coupling parameters $\xi_{\mathrm{c} 1}, T_{1}$, $\xi_{\mathrm{c} 2}$, and $T_{2}$. Theoretical analysis and numerical simulations have been carried out, and rich nonlinear dynamics have been observed in the system modeled by Eqs. (1)-(8). Complex chaotic dynamics are found in the system. The characteristics of a typical chaotic state are shown in Fig. 2. As is seen from these characteristics, the time series and power spectra of both lasers are demonstrated to develop into a chaotic state. Many interesting phenomena such as generation of chaos, quasiperiodic and period-doubling bifurcation to chaos, and death by delay are observed in semiconductor lasers with mutual coupling. Details of these phenomena are discussed in the following sections with experimental results.

In the experiments described in the following sections, the lasers are InGaAsP/InP single-mode DFB lasers both at $1.299 \mu \mathrm{m}$ wavelength. Both lasers are temperature stabilized at $21^{\circ} \mathrm{C}$. The photodetectors are InGaAs photodetectors with a $6 \mathrm{GHz}$ bandwidth, and the amplifiers are Avantek SSF86 amplifiers of $0.4-3 \mathrm{GHz}$ 

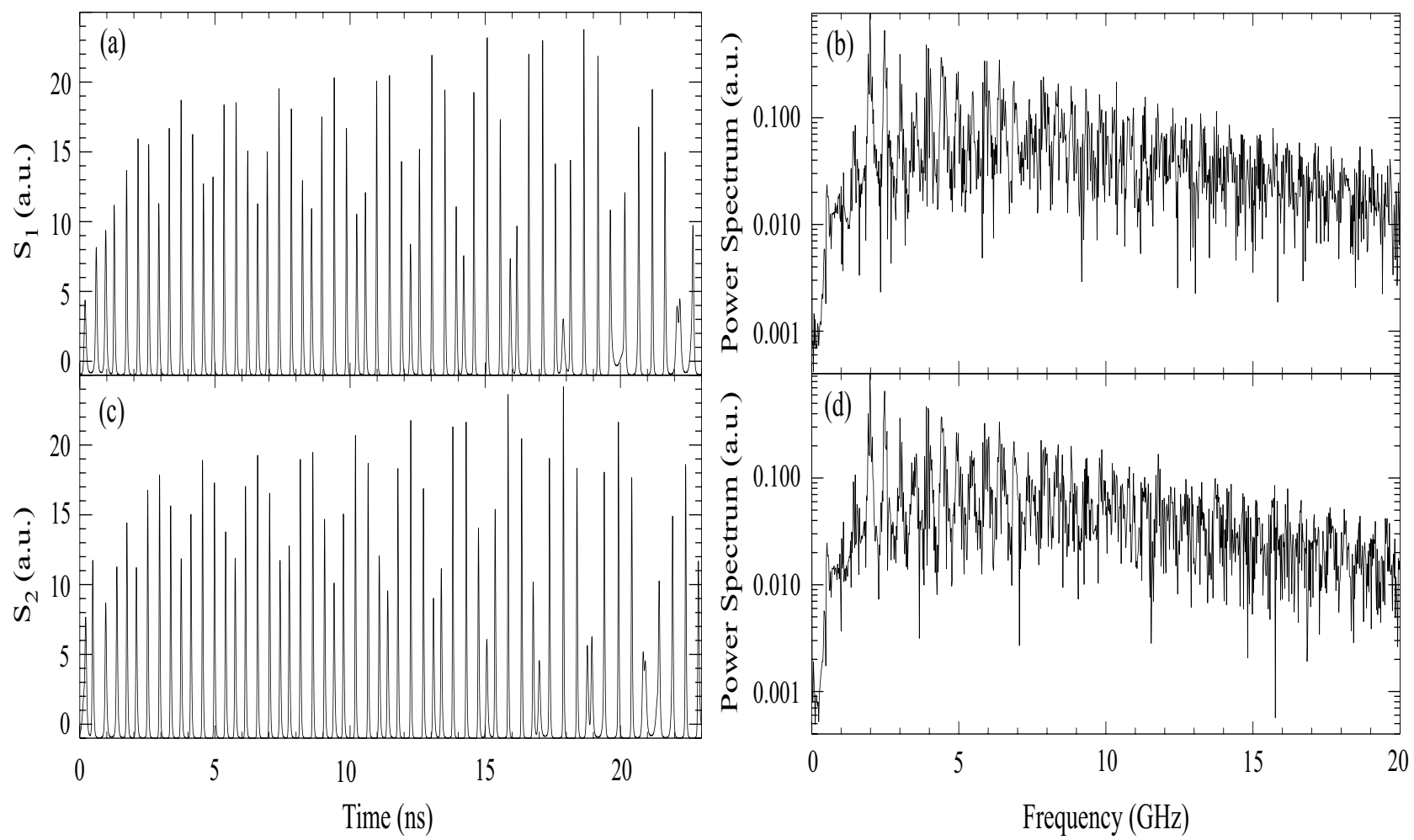

Figure 2. Numerically calculated time series of mutually coupled (a) LD1 and (c) LD2 showing fully developed chaotic oscillations. Corresponding power spectra are shown in (b) and (d).

bandpass. The intensity measured by photodetectors are recorded with a Tektronix TDS 694C digitizing sampling oscilloscope with a $3 \mathrm{GHz}$ bandwidth and up to $1 \times 10^{10}$ Samples/s sampling rate. Power spectra are measured with an HP E4407B RF spectrum analyzer that has a spectrum range from $9 \mathrm{kHz}$ to $26.5 \mathrm{GHz}$.

\section{MUTUAL COUPLING WITH NO FEEDBACK ON BOTH LASERS}

Setup 1 in Fig. 1 indicates the situation where the two semiconductor lasers have mutual optoelectronic coupling but no optoelectronic feedback. Without mutual coupling, the two lasers are solitary lasers. No complex nonlinear dynamics are observed in such solitary lasers. However, with mutual coupling, complex dynamics are observed in the system because its dynamical dimension is much increased by this delayed coupling mechanism. With Setup 1, complex dynamics such as chaos are observed when one or both lasers are biased below threshold. When both lasers are biased above threshold, no chaotic pulsing is observed. One route to chaos in this system is shown to be quasiperiodicity. In addition, period-doubling bifurcation is also found in this system when the coupling delay time or the coupling strength is varied.

Chaotic dynamics and a quasiperiodic route to chaos are observed in this system. Figure 3 shows a sequence of three dynamical states which are regular pulsing (RP), two-frequency quasiperiodic pulsing (Q2), and chaotic pulsing $(\mathrm{C})$, respectively, obtained by varying the coupling delay time $T_{2}$. For each dynamical state, the time series, power spectrum, and phase portrait from the system output of PD2 are plotted as in the first, second, and third columns, respectively. The output of the system from PD1 is similar to that from PD2 for each dynamical state.

In Figs. 3(a)-(c), the system is in a regular pulsing state. The time series in Fig. 3(a) shows a train of regular pulses with a constant pulsing intensity and interval. The corresponding power spectrum Fig. 3(b) has only one fundamental pulsing frequency, $f_{1}$, which is about $1 \mathrm{GHz}$. The phase portrait Fig. $3(\mathrm{c})$ is obtained by recording a 

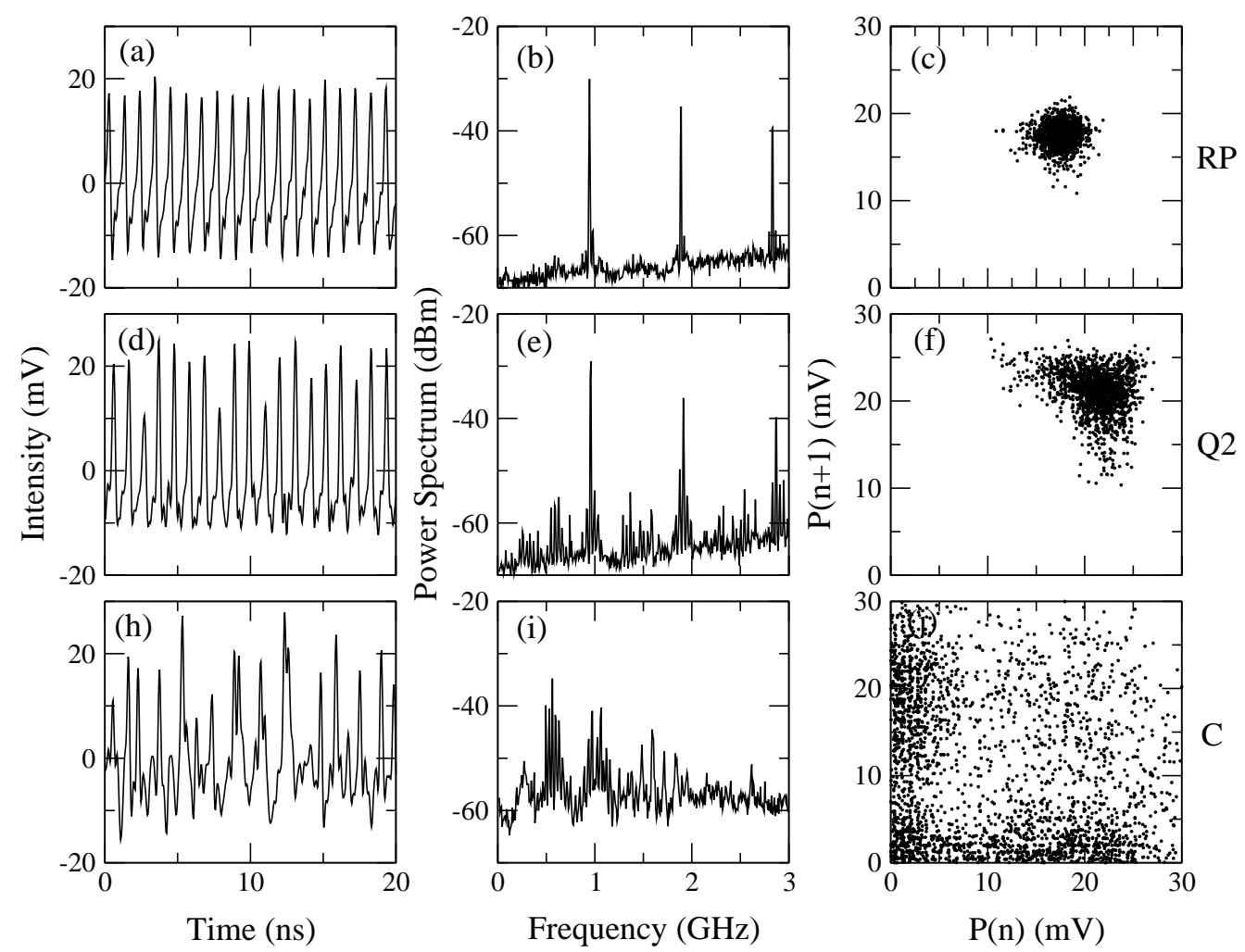

Figure 3. Quasiperiodic pulsing route to chaos for mutually coupled lasers with the configuration of Setup 1. RP: Regular pulsing state; Q2: Two-frequency quasiperiodic pulsing state; C: Chaotic pulsing state. First colum, time series; Second colum, power spectra; Third colum, phase portraits.

peak sequence $\mathrm{P}(\mathrm{n})$ at the local intensity maxima of a pulse train and further plotting $\mathrm{P}(\mathrm{n})$ versus $\mathrm{P}(\mathrm{n}+1)$. In the regular pulsing state, the output has a constant peak intensity, and the phase portrait shows only one spot. The fluctuations in the time series and, consequently, the scattering in the phase portrait are mainly caused by the noise in the system and the sampling errors from the oscilloscope. When the coupling delay time $T_{2}$ is decreased, the system enters a two-frequency quasiperiodic pulsing state with the pulsing intensity modulated at a certain frequency $f_{2}$ as shown in Figs. 3(d)-(f). The time series clearly shows the modulation of the peak intensity. In the power spectrum, except the pulsing frequency $f_{1}$, an incommensurate $f_{2}$ indicating the modulation of peak intensity shows up. This $f_{2}$ is related to the coupling delay time $T_{1}$ and $T_{2}$ of the mutual coupling loop because of the nonlinear interaction in this system. The appearance of two incommensurate frequencies, $f_{1}$ and $f_{2}$, is the indication of quasiperiodicity. In the phase portrait, the data points are still scattered due to noise and sampling errors. However, we can see that the distribution in Fig. 3(f) is more scattered than that in Fig. 3(c) because of the modulation on the pulse intensity. In Figs. 3(h)-(j), when $T_{2}$ is further decreased, the system enters a chaotic pulsing state. From the time series, we find that both the pulse intensity and the pulsing interval vary chaotically. At the same time, the power spectrum of the chaotic pulsing state is broadened with a much increased background, indicating the onset of chaos. The phase portrait shows a highly scattered distribution in a large area. Therefore, the system is shown to enter a chaotic pulsing state through a quasiperiodic route.

\section{MUTUAL COUPLING WITH FEEDBACK ON ONLY ONE LASER}

In Setup 1, the two lasers have only mutual coupling but no feedback. This setup can be modified to include a feedback loop on one laser, as is shown in Setup 2 of Fig. 1. In Setup 2, the output from LD1 is split 
into two parts. One is coupled to LD2, and the other is fed back to drive LD1 through an optoelectronic feedback loop that consists of PD1 and the corresponding amplifier. Therefore, even without mutual coupling, LD1 can have its own nonlinear dynamics due to the optoelectronic feedback. The dynamics of a semiconductor laser with optoelectronic feedback has been investigated, and a quasiperiodic route to chaos has been reported. ${ }^{3,4}$ Nevertheless, mutual coupling can further increase the complexity of both the system and its nonlinear dynamics.

Figure. 4 shows a sequence of dynamical states obtained with Setup 2 by varying the coupling delay times $T_{1}$ and $T_{2}$. From top to bottom, the total coupling delay time $T_{1}+T_{2}$ is gradually increased. A mixed bifurcation of quasiperiodicity and period doubling showing the route to chaos is demonstrated. In Fig. 4, the five rows show five dynamical states, which are regular pulsing (RP), quasiperiodic pulsing (Q2), period-two pulsing (P2), mix of period-two and quasiperiodic pulsing (P2+Q2), and finally chaotic pulsing $(\mathrm{C})$. For each dynamical state, the time series, power spectrum, and phase portrait from the system output of PD2 are plotted as in the first, second, and third columns, respectively. The output of the system from PD1 is similar to that from PD2 for each dynamical state.

The first two dynamical states are the RP and the Q2 states, which are similar to those in Fig. 3. The third state is a P2 state, where the pulse intensity has two distinct values which repeat one after another. In the power spectrum, besides the pulsing frequency $f_{1}$, the subharmonic of $f_{1} / 2$ also shows up. The other frequency peaks are the harmonics and combinations of $f_{1}$ and $f_{1} / 2$. In the phase portrait, two scattered spots are clearly observed, which is the characteristic of a period-two state. From the P2 state, the system further evolves into a mixed state of P2 and Q2. In the time series, a modulation on the pulse intensity is observed. In the power spectrum, a second fundamental frequency $f_{2}$ shows up besides the frequency peaks of a typical P2 state. The phase portrait shows two spots with more scattering than those two spots in a simple P2 state due to the modulation on the peak intensity. Finally, the system enters a chaotic pulsing state. Therefore, it is demonstrated that a mixed bifurcation of quasiperiodicity and period-doubling coexists in this system.

With Setup 2 as shown in Fig. 1, besides the mutual coupling between the two lasers, one of the lasers also has an optoelectronic feedback loop. Without mutual coupling, one laser still has its own nonlinear dynamics. The mutual coupling further increases the dynamical dimension of the system which results in highly complex dynamics in the system. A mixed bifurcation of quasiperiodicity and period-doubling is demonstrated. The system can get into chaos through a quasiperiodic pulsing route or a period-doubling route or a mixture of these two. The system with Setup 2 is found to be prone to chaos, and such chaos is more complex than that found in the system with Setup 1. Since LD1 can have nonlinear dynamics before mutual coupling, an interesting phenomenon of death by delay is also observed in this setup. In the death by delay phenomenon, LD1 is operated in a pulsing state before mutual coupling. After mutual coupling, this oscillation is quenched to zero amplitude due to the delayed coupling. Details of death by delay are further discussed in the following section.

\section{MUTUAL COUPLING WITH FEEDBACK ON BOTH LASERS}

In Setup 3 as shown in Fig. 1, each of the two mutually coupled lasers also has an optoelectronic feedback loop of its own. Even without mutual coupling, both LD1 and LD2 can have their own nonlinear dynamics due to their optoelectronic feedback. The two lasers with their optoelectronic feedback are two nonlinear oscillators. With this setup, we can study the coupling effect between two nonlinear oscillators. The coupling effect is found to be very significant. Depending on the coupling delay time and coupling strength, the mutual coupling can either stabilize the nonlinear oscillation as in a death-by-delay phenomenon, or it can drive the nonlinear oscillation into more complex dynamical states such as highly chaotic oscillations.

Death by delay is a very interesting and important phenomenon where two limit-cycle oscillators suddenly stop oscillating due to a time-delayed coupling between the two oscillators. This phenomenon has been theoretically predicted in coupled oscillators and has been experimentally observed in coupled circuits and biological systems. $^{7-9}$ The investigation on death by delay is important because the world is full of oscillators which are often coupled together. A sudden death of some oscillations can have serious consequences. Using Setup 3 in Fig. 1, we can conveniently study the effect of death by delay in semiconductor laser oscillators in particular and the effect in general. The mutual coupling strength and the coupling delay time can be adjusted by changing the attenuation on the coupled optical power and the optical path length in the coupling channel, respectively. 

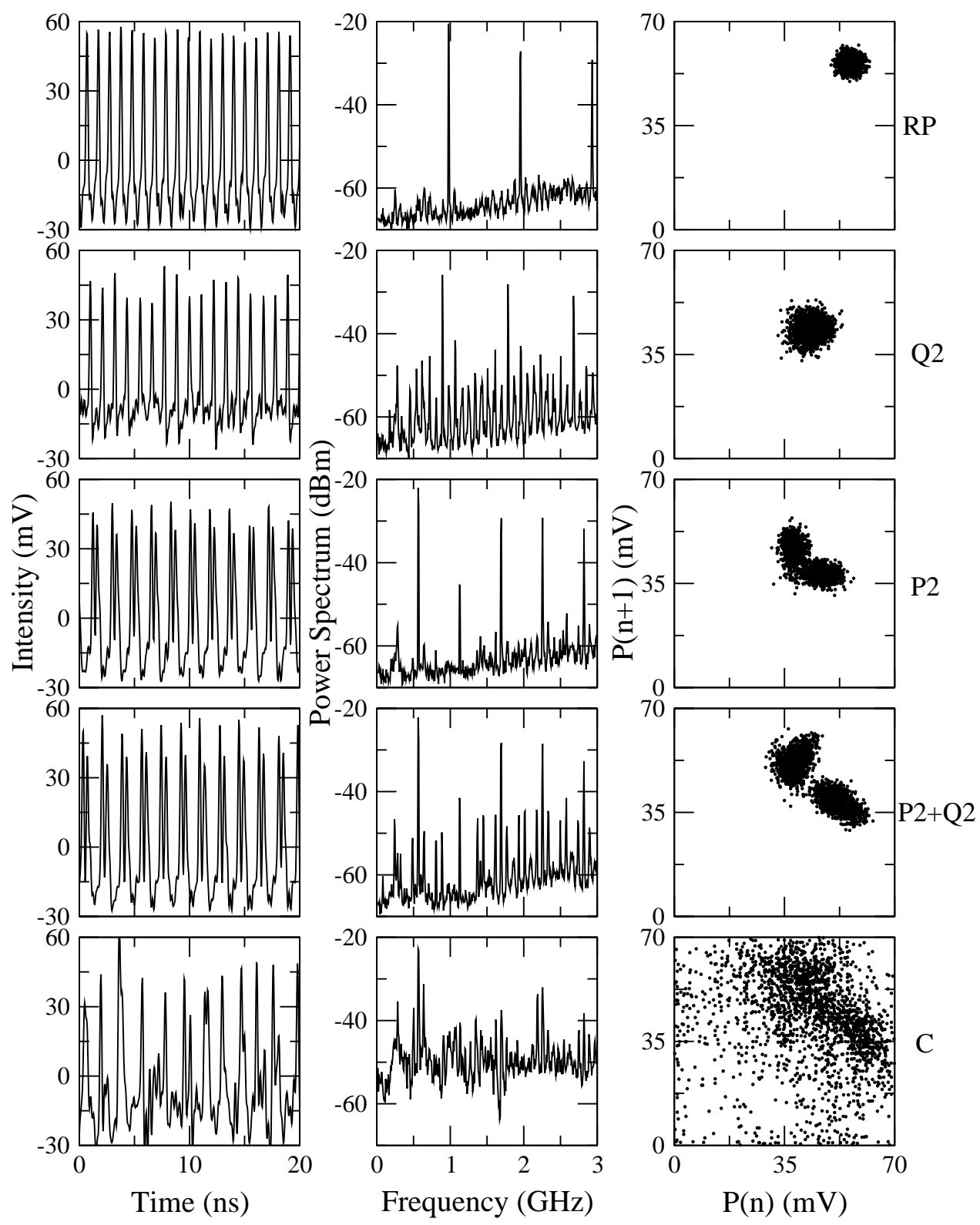

Figure 4. Mixed quasiperiodic and period-doubling bifurcation to chaos for mutually coupled lasers with the configuration of Setup 2. RP: Regular pulsing state; Q2: Two-frequency quasiperiodic pulsing state; P2: Period-doubling state; P2+Q2: Mixture of period-doubling and two-frequency quasiperiodic state; C: Chaotic pulsing state. First colum, time series; Second colum, power spectra; Third colum, phase portraits.

The phenomenon of death by delay is observed in the coupled semiconductor lasers with very high frequency limit-cycle oscillations. Figure 5 shows the time series, power spectra, and correlation plots of the outputs from PD1 and PD2 in the setup before and after mutual coupling. Before mutual coupling, the two lasers are operated in independent limit-cycle oscillations. The time series and the power spectra of such oscillations are shown in Figs. 5(a) and (b), respectively. As is shown, the two lasers oscillate at regular pulsing states with almost the same fundamental frequency at $1 \mathrm{GHz}$. However, because the two oscillations are independent and their 

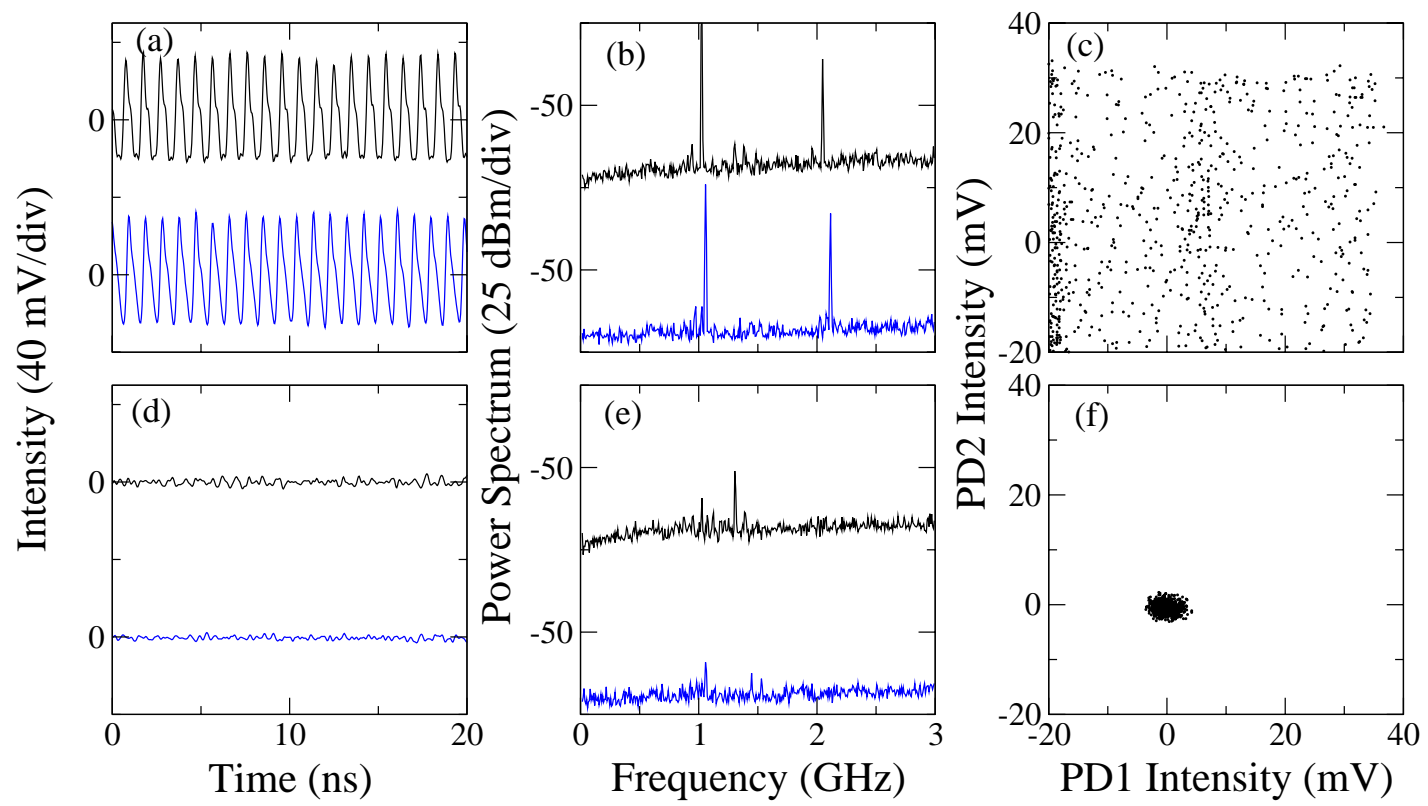

Figure 5. Phenomenon of death by delay for mutually coupled lasers with the configuration of Setup 3. (a)-(c), time series, power spectrum, and correlation plot of the outputs from PD1 and PD2 before mutual coupling. (d)-(f), corresponding plots after mutual coupling.

oscillation frequencies are not exactly the same, the output from the two lasers are not correlated. Figure 5(c) shows the correlation plot of the output from PD1 versus that from PD2. The correlation plot is scattered all over the place, which indicates that the two lasers oscillate with uncorrelated large amplitude variations. However, as soon as mutual coupling is applied to the two semiconductor lasers, the original large-amplitude oscillations are suddenly quenched to almost zero amplitudes. The time series and the power spectra of the outputs in this state of mutual coupling are shown in Figs. 5(d) and (e), respectively. As is shown, no oscillations exist anymore, and the amplitudes of both time series are almost zero. The flat spectra in Fig. 5(e) are close to the noise floor. The residual fluctuations in the two lasers after mutual coupling are caused by noise from the lasers and the electronic circuits in the system. Nevertheless, comparing the time series and the power spectra before and after mutual coupling, it is clear that the oscillations are almost completely quenched by the mutual coupling between the two oscillators. Figure 5(f) shows the correlation plot which is almost a single spot due to the quenched amplitude of the oscillations. In the plots of time series and power spectra, the output from PD2 is down shifted for clear comparison.

The phenomenon of death by delay happens only at certain coupling delay times. As the two lasers are mutually coupled to each other through separate paths, the mutual coupling delay times $T_{1}$ and $T_{2}$ can be adjusted separately. However, it is the total mutual coupling delay time $T_{1}+T_{2}$ that determines the appearance of a death island. Multiple death islands are observed when the total mutual coupling delay time $T_{1}+T_{2}$ is changed over a wide range. Figure 6 shows four death islands when $T_{1}+T_{2}$ is varied. In Fig. 6 , the circles with the connecting bars show the death islands when $T_{2}$ is changed first, and the squares with the connecting bars show the death islands when $T_{1}$ is changed first. When the total coupling delay time is increased, a sequence of death islands show up at multiple positions which have almost the same separations. In Fig. 6 the averaged separation between the islands is about $1 \mathrm{~ns}$. This time separation matches with the inverse of the original oscillation frequency before coupling, which is about $1 \mathrm{GHz}$ as shown in Fig. 5(b). When we either change $T_{1}$ first or $T_{2}$ first, the values of $T_{1}$ and $T_{2}$ are not the same for each death island. However, the total values of $T_{1}+T_{2}$ are the same for each corresponding death island no matter whether $T_{1}$ or $T_{2}$ is changed. This observation confirms that the appearance of a death island really depends on the total $T_{1}+T_{2}$ but not the separate values 


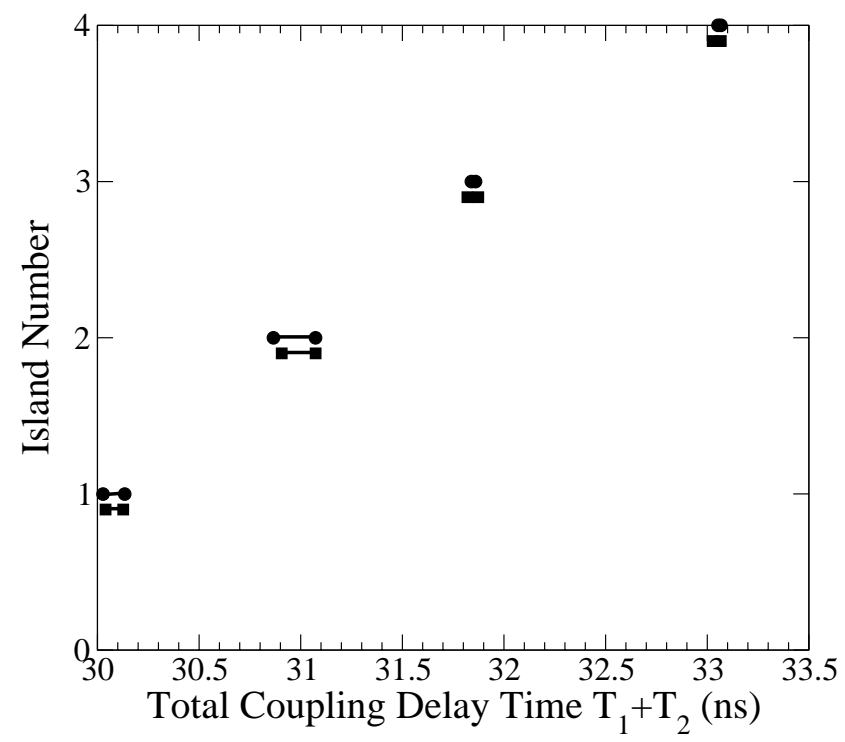

Figure 6. Total coupling delay time $T_{1}+T_{2}$ for multiple death islands.

of $T_{1}$ or $T_{2}$.

As we have discussed in Section 4, the phenomenon of death by delay is also observed in Setup 2 as shown in Fig. 1 where only one laser has optoelectronic feedback. Actually, the locations of the death islands for Setup 2 and Setup 3 are matched at the same values of $T_{1}+T_{2}$. Based on our investigations, the phenomenon of death by delay is caused by a negative feedback loop from the mutual coupling to each oscillator, which quenches the original oscillation. The dependence of the death islands on the total mutual coupling delay time confirms our explanation because the total mutual coupling delay time is exactly the delay time in this negative feedback loop created by the mutual coupling. Since the phase shift repeats after every period of the oscillation, the death islands can be repeated when the total mutual coupling delay time is changed by a period of the oscillation. This is in good agreement with the experimental results presented in Fig. 6.

As is shown in Fig. 5, mutual coupling between two oscillators can quench the oscillation amplitude and stabilize the coupled semiconductor lasers. On the other hand, mutual coupling can also drive the coupled oscillators into chaotic states and destabilize the coupled semiconductor lasers. The chaotic state and the route to chaos are similar to those obtained with Setups 1 and 2. A mixed bifurcation of quasiperiodicity and perioddoubling is observed.

\section{DISCUSSIONS}

From Setup 1 to Setup 3, the system gradually changes from mutually coupled semiconductor lasers without feedback, to one laser with feedback, and eventually to both lasers with feedback. We can also compare the dynamical states when such change in configuration evolves. The dynamics change significantly when the configuration is changed. With the complete Setup 3 in Fig. 1, we can conveniently block the mutual coupling, any one of the two feedback loops, or both of the feedback loops. Thus we can study changes in the dynamical states of the system with the same operating conditions but under different configurations with or without mutually coupling or feedback. Figure 7 shows a sequence of dynamical states when all the operating conditions are fixed but with the mutual coupling or feedback channel blocked in different ways. For each situation, the time series, correlation plot, and correlation coefficient are plotted in the first, second, and third columns, respectively.

In the first situation as shown in row (a), the two lasers both have their own optoelectronic feedback but with no mutual coupling. As is shown, the two lasers each oscillates at an independent regular pulsing state due 

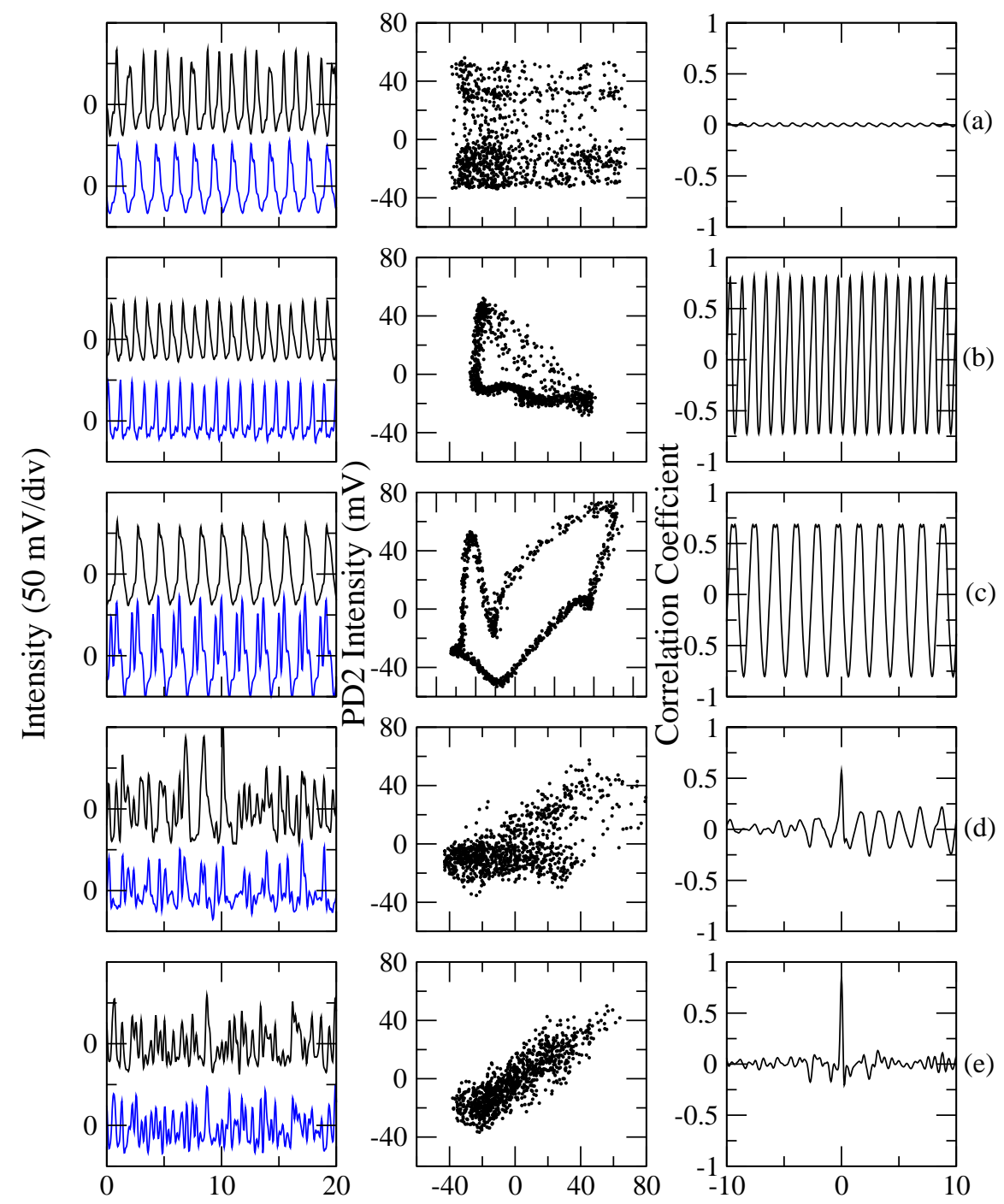

Time (ns)

PD1 Intensity (mV)
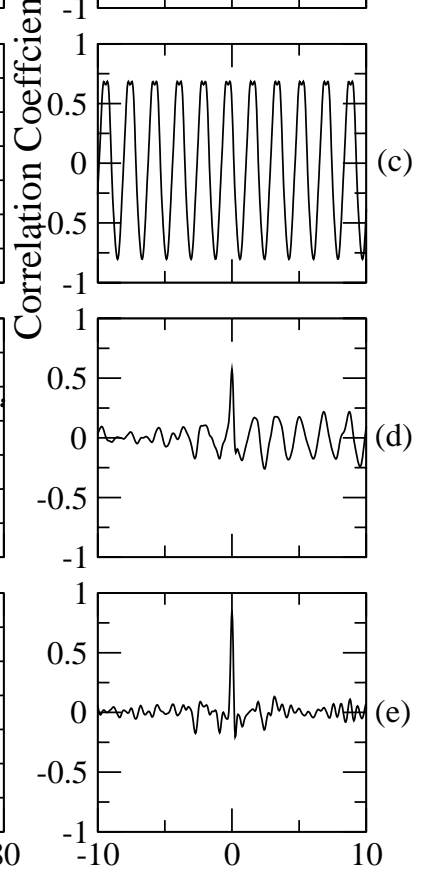

Time Shift (ns)

Figure 7. Different dynamical states under the same operating conditions but with different configurations in the setup. Row (a), LD1 and LD2 both have optoelectronic feedback but with no mutual coupling; Row (b), LD1 and LD2 have mutual coupling but with no optoelectronic feedback; Row (c), LD1 and LD2 have mutual coupling but only LD2 has optoelectronic feedback; Row (d), LD1 and LD2 have mutual coupling but only LD1 has optoelectronic feedback; Row (e), LD1 and LD2 have mutual coupling and both have optoelectronic feedback. First colum, time series; Second colum, correlation plot of output from PD1 vs. that from PD2; Third colum: shifted correlation coefficient between the outputs from PD1 and PD2.

to the feedback effect. However, the two outputs are not correlated because there is no mutual coupling between them. Thus the correlation plot shows a very scattered distribution over the entire area. Shifted correlation coefficient is calculated between the outputs from PD1 and PD2 when the waveform from PD2 is time shifted gradually. As is shown, there is no correlation peak in the plot which indicates that indeed the two lasers are not correlated when they are not mutually coupled. In the second situation as shown in row (b), the two lasers are mutually coupled but none of them has a feedback loop. With mutual coupling only, the two lasers can 
have some nonlinear dynamics due to the nonlinear effect from mutual coupling. Under the current operating conditions, the two lasers oscillate in regular pulsing states. Different from the first situation, now the two lasers are mutually coupled and thus their oscillations are correlated. As is shown, the two lasers oscillate at the same pulsing frequency. The correlation plot shows a simple pattern which indicates that the two outputs are correlated with some phase shift. In the shifted correlation coefficient, there are oscillating correlation peaks which means that the two waveforms are correlated regular pulsing waveforms. The correlation coefficient repeats when the waveform from PD2 is phase shifted by $2 \pi$ every time. In the third situation as shown in row (c), the two lasers have mutual coupling between them but only LD2 has optoelectronic feedback. The two lasers are not symmetric under this situation because one laser has feedback while the other one does not. As a result, the outputs from PD1 and PD2 are not exactly the same but are phase correlated. The output from PD1 is a regular pulsing state, while that from PD2 is a period-two pulsing state. The correlation plot shows that the two waveforms are correlated with fixed phase shift. The correlation coefficient also has high peaks which oscillate due to the simple waveform pattern. In the fourth situation shown in row (d), the two lasers have mutual coupling between them but only LD1 has optoelectronic feedback. Although there are mutual coupling in both situations (c) and (d), the laser which has optoelectronic feedback is switched in those two situations. Since the two lasers can have slightly different intrinsic and operating feedback parameters, the dynamical states are different in (c) and (d). In row (d), the outputs from PD1 and PD2 are chaotic due to the combined effect of mutual coupling and feedback. The two waveforms are partially correlated because the lasers are mutually coupled. The shifted correlation coefficient also shows a correlation peak at the center. However, this correlation is not very high because the two lasers are not symmetric. Finally, in the fifth situation as shown in row (e), the two lasers have mutual coupling between them and they both have optoelectronic feedback also. Thus the two lasers are exactly symmetric in the configuration. As is shown, the outputs are highly complex chaotic waveforms due to the combined effect of mutual coupling and optoelectronic feedback. Furthermore, the two chaotic waveforms are highly correlated with a very nice distribution along the $45^{\circ}$ diagonal, indicating that the two lasers are reliably synchronized. The shifted correlation coefficient also has a single peak at zero time shift which means that the two waveforms are synchronized in time. The high quality of synchronization between the two outputs are due to the mutual coupling and the symmetric configuration between the two lasers.

Thus in Fig. 7 from (a) to (e), the complexity of the system configuration is gradually increased as the dynamics evolve from simple regular pulsing state to chaotic pulsing state. Although the complexity of the dynamical states is increased, the correlation property between the two outputs is also increased due to the mutual coupling between the two lasers and also the increased symmetric configuration between them.

\section{CONCLUSIONS}

Mutually-coupled semiconductor lasers are investigated in order to study the effect of mutual coupling on semiconductor lasers in particular and such effect on mutually coupled systems in general. It is found that mutual coupling can significantly affect the dynamical states of the semiconductor lasers, depending on the coupling delay time and the coupling strength. Highly complex chaos can be generated in the mutually coupled lasers. A mixed bifurcation of quasiperiodicity and period-doubling is found in such lasers. Death by delay caused by the effect of mutual coupling is demonstrated. High quality of synchronization between the chaotic outputs from mutually coupled semiconductor lasers are also observed. From these experimental demonstrations, it is shown that mutual coupling plays very important roles in coupled dynamical systems.

\section{ACKNOWLEDGMENTS}

This work is supported by the U.S. Army Research Office under contract No. DAAG55-98-1-0269.

\section{REFERENCES}

1. "Special issue on applications of chaos in modern communication systems," IEEE Trans. Circuits Syst. I, vol. 48 , Dec. 2001.

2. "Feature section on optical chaos and application to cryptography," IEEE J. Quantum Electron., vol. 38, Sept. 2002. 
3. S. Tang and J. M. Liu, "Chaotic pulsing and quasiperiodic route to chaos in a semiconductor laser with delayed optoelectronic feedback," IEEE J. Quantum Electron., vol. 37, no. 3, pp. 329-336, Mar. 2001.

4. F.Y. Lin and J.M. Liu, "Nonlinear dynamics of a semiconductor laser with delayed negative optoelectronic feedback," IEEE J. Quantum Electron., vol. 39, pp.562-568, Apr. 2003.

5. T. Heil, I. Fischer, W. Elsasser, J. Mulet, and C.R. Mirasso, "Chaos synchronization and spontaneous symmetry-breaking in symmetrically delay-coupled semiconductor lasers," Phys. Rev. Lett., vol. 86, pp. 795798, Jan. 2001.

6. J. Mulet, C. Masoller, and C.R. Mirasso, "Modeling bidirectionally coupled single-mode semiconductor lasers," Phys. Rev. A, vol. 65, Art. No. 063815, June 2002.

7. D.V.R. Reddy, A. Sen, and G.L. Johnston, "Experimental evidence of time-delay-induced death in coupled limit-cycle oscillators," Phys. Rev. Lett., vol. 85, pp. 3381-3384, Oct. 2000.

8. A. Takamatsu, T. Fujii, and I. Endo, "Time delay effect in a living coupled oscillator system with the plasmodium of Physarum polycephalum," Phys. Rev. Lett., vol. 85, pp. 2026-2029, Aug. 2000.

9. R. Herrero, M. Figueras, J. Rius, F. Pi, and G. Orriols, "Experimental observation of the amplitude death effect in two coupled nonlinear oscillators," Phys. Rev. Lett., vol. 84, pp. 5312-5315, June 2000. 